\title{
Spatial Distribution of Single-Nucleotide Polymorphisms Related to Fungicide Resistance and Implications for Sampling
}

\author{
H. Van der Heyden, P. Dutilleul, L. Brodeur, and O. Carisse
}

First and second authors: Department of Plant Science, McGill University, Macdonald Campus, 21,111 Lakeshore Road, Ste-Anne-deBellevue, Quebec H9X 3V9, Canada; first and third authors: Compagnie de Recherche Phytodata Inc., 111 Rang Saint-Patrice, Sherrington, Quebec JOL 2NO, Canada; and first and fourth authors: Agriculture and Agri-Food Canada, 430 Boulevard Gouin, St-Jeansur-Richelieu, Quebec J3B 3E6, Canada.

Accepted for publication 20 December 2013.

\begin{abstract}
Van der Heyden, H., Dutilleul, P., Brodeur, L., and Carisse, O. 2014. Spatial distribution of single-nucleotide polymorphisms related to fungicide resistance and implications for sampling. Phytopathology 104:604-613.

Spatial distribution of single-nucleotide polymorphisms (SNPs) related to fungicide resistance was studied for Botrytis cinerea populations in vineyards and for $B$. squamosa populations in onion fields. Heterogeneity in this distribution was characterized by performing geostatistical analyses based on semivariograms and through the fitting of discrete probability distributions. Two SNPs known to be responsible for boscalid resistance (H272R and H272Y), both located on the B subunit of the succinate dehydrogenase gene, and one SNP known to be responsible for dicarboximide resistance (I365S) were chosen for B. cinerea in grape. For $B$. squamosa in onion, one SNP responsible for dicarboximide resistance (I365S homologous) was chosen. One onion field was sampled in 2009 and another one was sampled in 2010 for $B$. squamosa, and two vineyards

were collected and analyzed by restriction fragment length polymorphism polymerase chain reaction (PCR) or allele specific PCR. Mean SNP incidence varied from 16 to $68 \%$, with an overall mean incidence of $43 \%$. In the geostatistical analyses, omnidirectional variograms showed spatial autocorrelation characterized by ranges of 21 to $1 \mathrm{~m}$. Various levels of anisotropy were detected, however, with variograms computed in four directions (at $0^{\circ}, 45^{\circ}, 90^{\circ}$, and $135^{\circ}$ from the within-row direction used as reference), indicating that spatial autocorrelation was prevalent or characterized by a longer range in one direction. For all eight data sets, the $\beta$-binomial distribution was found to fit the data better than the binomial distribution. This indicates local aggregation of fungicide resistance among sampling units, as supported by estimates of the parameter $\theta$ of the $\beta$-binomial distribution of 0.09 to 0.23 (overall median value $=0.20$ ). On the basis of the observed spatial distribution patterns of SNP incidence, sampling curves were computed for different levels of reliability, emphasizing the importance of sample size for the detection of mutation incidence below the risk threshold for control failure.
\end{abstract} were sampled in 2011 for $B$. cinerea, for a total of four sampled sites. Cluster sampling was carried on a 10-by-10 grid, each of the 100 nodes being the center of a 10-by-10-m quadrat. In each quadrat, 10 samples
Additional keywords: spatial heterogeneity.

In the early 1990s, Gareth Hughes and Laurence Madden introduced the use of discrete probability distributions to characterize spatial heterogeneity in plant disease epidemiology $(16,17$, $26,27,30,32)$. In this approach, the number of diseased plants among $n$ plants randomly sampled in a quadrat, for example, is assumed to follow a binomial distribution for a completely random spatial pattern and a $\beta$-binomial distribution for an aggregated spatial pattern. In fact, one of the parameters $(\theta)$ of the betabinomial distribution is a direct measurement of local aggregation. These two discrete probability distributions have been widely used to characterize homogeneity versus heterogeneity of plant disease epidemics for soilborne pathogens and airborne pathogens and viruses. For example, Xiao et al. (52) found that Verticillium dahlia microsclerotia in soil were aggregated; in strawberry fields, Turechek et al. $(49,50)$ showed that leaf blight and leaf spot diseases tended to be aggregated; and, for viruses, Dallot et al. (9) recently discovered aggregation in Shraka disease, caused by the Plum pox virus. The level of spatial aggregation obtained in these studies was used, in part, to develop effective sampling strategies, which helped determine disease level and improve timing of control measure application.

Even though many integrated management strategies have been developed to delay plant disease epidemics, disease control continues to depend mostly on fungicide applications. An important factor for an efficient fungicide application is the proportion of resistant individuals present in the target population (4). Despite the importance of the spatial distribution of fungicide resistance
This article is in the public domain and not copyrightable. It may be freely reprinted with customary crediting of the source. The American Phytopathological Society, 2014 
(to be distinguished from the spatial distribution of a plant pathogen), most of the epidemiological studies of fungicide resistance have been targeted on temporal changes, such as the increase in resistance frequency following consecutive fungicide applications $(34,35,37)$. In the late 1990s, Elmer et al. (11) studied the spatial component of dicarboximide resistance in Monilinia fructicola populations on stone fruit. The results obtained for stone fruit suggested various levels of heterogeneity in the spatial distribution of dicarboximide-resistant strains of M. fructicola populations. Invasion of a resistant strain over a sensitive one mainly follows from the difference in fitness between the two strains as well as the resistance factor associated with the resistant phenotype.

In the last decade, breakthroughs at the individual level have been made in the identification of molecular mechanisms related to fungicide resistance. For the model organism Botrytis cinerea, responsible for diseases of $>225$ hosts, single-nucleotide polymorphisms (SNPs) have been identified in both field and lab mutants and related to resistant phenotypes for at least six fungicide groups $(1,2,7,8,13,14,24,25,38,39,46,47,54)$. Ranges of resistance factor and impact on pathogen fitness were also determined for each of these SNPs. In addition, the results of Van der Heyden et al. (unpublished data) suggest that patterns of spatial co-existence are also possible at the SNP scale and that a genetic niche occupied by a specific SNP might either prevent invasion by or coexistence with other SNPs within a given population.

Therefore, breaking down the phenotypic resistance into genotypic factors (i.e., SNPs) is of great practical importance for monitoring fungicide resistance and foreseeing shifts in resistant populations. Considering the value of knowing the frequency of resistant strains carrying a particular SNP in a given population, the question of sampling naturally arises in this approach. Few studies on fungicide resistance have focused on spatial aspects, and we could not find any studies in which the spatial distribution patterns of SNPs related to fungicide resistance were investigated (11). Hence, knowing the level of local aggregation is essential to making accurate inference and determining appropriate sample size. Accordingly, by using the geostatistical and distributional approaches, the objectives of this study were to (i) formally characterize the spatial distribution pattern of three SNPs related to fungicide resistance to boscalid and iprodione within $B$. cinerea populations in vineyards, (ii) do the same characterization for one SNP related to fungicide resistance to iprodione within $B$. squamosa populations in onion fields, and (iii) compute sampling curves relative to the estimation of SNP frequency for various levels of spatial aggregation.

\section{MATERIALS AND METHODS}

Sampling protocols. Sampling for $B$. cinerea was conducted during fall 2011 in two commercial vineyards (of 9 and 4.5 ha, respectively), each one planted with 'Seyval' grape cultivar and located in the Eastern Townships region of the Province of Québec (Canada). In both vineyards, sampling was organized following a 100-by-100-m grid installed in the middle of the field and divided in 100 10-by-10-m quadrats. At the center of each quadrat, spores from 10 single-sporulating colonies were individually sampled in duplicate, using a dry BBL culture swab (Fisher Scientific, Ottawa, ON, Canada). Once in the lab, culture swabs were placed into a 2-ml skirted screw-cap micro-test tube, containing $380 \mu \mathrm{l}$ of isopropanol (Sigma-Aldrich Canada Ltd., Oakville, ON, Canada). Each sample was placed at $-20^{\circ} \mathrm{C}$ until upstream polymerase chain reaction (PCR) analysis.

Sampling for B. squamosa was conducted in commercial onion fields in a muck land area near Montréal (Québec, Canada) during fall 2009 and 2010. In 2009, 'Red Bull' onion was seeded on 23 April 23 at the rate of 30 seeds $\mathrm{m}^{-1}$, with row spacing of $0.45 \mathrm{~m}$ in a 6-ha field; in 2010, 'Hamlet' onion was seeded on 26 April at the same rate and row spacing in a 12-ha field. Sites were different in the 2 years but, at each site, sampling was organized in the middle of the field following a 100-by-100-m grid divided in 100 10-by-10-m quadrats. At the center of each quadrat, 10 infected leaves were randomly collected on different onion plants and kept on ice, prior to being brought to the lab by the end of the day. Each leaf was cut into $2-\mathrm{cm}$ pieces and placed in a 100-by$15-\mathrm{mm}$ petri dish with a sheet of $90-\mathrm{mm}$ Whatman filter paper amended with $500 \mu \mathrm{l}$ of milliQ water (Fisher Scientific). The petri dishes (10 per quadrat) were placed at room temperature (22 to $25^{\circ} \mathrm{C}$ ) for 2 to 5 days. In each petri dish, spores from singlesporulating lesions were harvested using a cotton swab drenched with isopropanol (Sigma-Aldrich Canada Ltd.) and placed into a 2-ml skirted screw-cap micro-test tube containing $300 \mu \mathrm{l}$ of isopropanol (Sigma-Aldrich Canada Ltd.). All samples were kept at $-20^{\circ} \mathrm{C}$ before DNA extraction and PCR analysis.

DNA extraction and restriction fragment length polymorphism PCR. To obtain total DNA, $80 \mu$ of spore suspension was transferred to a sterile 2-ml screw-cap micro-test tube containing $100 \mathrm{mg}$ of glass beads (425 to $600 \mu \mathrm{m}$ ) (Sigma-Aldrich Canada Ltd.). Tubes were shaken in a FastPrep-24 instrument (MP Biomedicals, Irvine, CA) at $4 \mathrm{~m} / \mathrm{s}$ for $20 \mathrm{~s}$ to fragilize the conidia cell wall and promote membrane disruption. Conidia were precipitated by centrifugation for $5 \mathrm{~s}$ at $10,000 \times g$, and isopropanol was evaporated under vacuum using a SpeedVac (Savant Instruments, Inc., Holbrook, NY) instrument for $20 \mathrm{~min}$ at $55^{\circ} \mathrm{C}$. Conidia were resuspended in $300 \mu$ l of DNA extraction buffer, consisting of nuclease-free water (Integrated DNA Technologies, Inc., Coralville, IA) and 5\% chelex-100 molecular-biology grade resin (Bio-Rad Laboratories, Hercules, CA). Following resuspension, tubes were placed in a dry bath at $105^{\circ} \mathrm{C}$ for $15 \mathrm{~min}$ to complete cell disruption and DNA extraction. Tubes were finally agitated for $5 \mathrm{~s}$, using a table vortex first and then a centrifugation at $15,000 \times g$ for $5 \mathrm{~min}$ at $4^{\circ} \mathrm{C}$. Supernatant containing genomic DNA was placed at $4^{\circ} \mathrm{C}$ until upstream PCR analysis for a maximum of $2 \mathrm{~h}$ or placed at $-20^{\circ} \mathrm{C}$ until future use.

Detection of the $\mathrm{I} 365 \mathrm{~S}$ allele in $B$. cinerea was performed using a PCR restriction fragment length polymorphism (RFLP) assay, as described by Oshima et al. (38), and that of the H272R and $\mathrm{H} 272 \mathrm{Y}$ alleles was completed with the procedure described by Yin et al. (53). Similarly, identification of the I365S allele in $B$. squamosa was performed in a PCR-RFLP assay. The primer pair BsqdxF (5'-CAGATATTCTCGAGTGAGGTCTCC-3') and BsqdxR (5'-CTCTGCCTGAACCTTCTGTGTCAA-3') was used for amplification of a 560-bp fragment. The PCR reactions were conducted in a $25-\mu$ l reaction volume containing $3 \mu \mathrm{l}$ of DNA extraction, $2.5 \mu \mathrm{l}$ of PCR buffer $(10 \times), 2 \mu \mathrm{l}$ of bovine serum albumen $\left(10 \mathrm{mg} \mathrm{ml}^{-1}\right), 0.25 \mu \mathrm{l}$ of dNTPs $(20 \mathrm{mM}), 0.38 \mu \mathrm{l}$ of each primer $(10 \mu \mathrm{M})$, and $0.5 \mu \mathrm{l}$ of Titanium Taq polymerase (Clontech Laboratories, Mountain View, CA). The amplification was carried in a SureCycler 8800 (Agilent, La Jolla, CA), with conditions set as follows: first, $95^{\circ} \mathrm{C}$ for $5 \mathrm{~min}$; then, 35 cycles of $30 \mathrm{~s}$ at $95^{\circ} \mathrm{C}$, $45 \mathrm{~s}$ at $62^{\circ} \mathrm{C}$, and $30 \mathrm{~s}$ at $72^{\circ} \mathrm{C}$; and, finally, $10 \mathrm{~min}$ at $72^{\circ} \mathrm{C}$.

The PCR products amplified by the primer pairs BcOS10R/ $\mathrm{BcOS} 5 \mathrm{a}$ and $\mathrm{BsqdxF} / \mathrm{R}$ were digested with the enzyme Taq $\alpha \mathrm{I}$, as recommended by the manufacturer. Digested products were separated by electrophoresis in $1.7 \%$ agarose gel in $1 \times$ Tris-acetate EDTA buffer and visualized after ethidium bromide staining under UV light.

Geostatistical analyses. Even though geostatistics were originally developed for continuous quantitative data, the semivariance was computed here on discrete quantitative data and was considered to be a reasonable measure of spatial autocorrelation in these conditions. Initially, maps of SNP incidence were produced from the number (from 0 to 10) of samples tested positive for the presence of a given SNP at the 100 spatially referenced quadrats on a 100-by-100-m sampling grid; these count data were assigned to the centers of quadrats. Then, the first phase of the method of 
coregionalization analysis with a drift (CRAD) was performed to detect and remove any possible drift (i.e., large-scale component of the spatial pattern) (44). In field phytopathological studies, it is expected that a drift includes the spatial variation that is not attributed to the intrinsic spatial behavior of the studied pathogen; for example, the presence of a slope would be incorporated into a drift. In this initial geostatistical analysis, the count data collected on a grid for a given SNP were considered as a partial realization of a random function $(Z)$, and their spatial pattern was decomposed into an estimated drift $(\hat{d})$ plus a residual component $(\hat{e})$ :

$$
Z\left(u_{i}\right)=(\hat{d})\left(u_{i}\right)+(\hat{e})\left(u_{i}\right)(i=1, \ldots, 100)
$$

where $u_{i}$ represents quadrat $i$. The drift was estimated with the estimated generalized least squares (EGLS) method, performed in a local procedure $\left(\mathrm{L}_{1}\right)$, including the selection of an optimal moving window size. Our CRAD analyses were carried out with the freeware available at http://environmetricslab.mcgill.ca/ Programs.html.

Second, to assess the presence of spatial autocorrelation in the count data, omnidirectional and directional experimental variograms were computed on residuals $\left(\hat{e}_{1}\right)$ and analyzed using PROC VARIOGRAM of SAS (version 9.3; SAS Institute, Cary, NC). Eight equi-frequent distance classes were used to compute the variograms; their boundaries were 10, 14.14, 20, 22.36, 30.36, $36.06,41.16$, and $44.72 \mathrm{~m}$. There were at least 150 pairs of observations in each distance class, which assured a good level of precision in the estimation of semivariance values. Matheron's classical semivariance estimator (33) was used, which for omnidirectional variograms is given by:

$$
\hat{\gamma}(h)=\frac{1}{2 A(h)} \sum_{\left(i, i^{\prime}\right)}\left(\hat{e}\left(u_{i}\right)-\hat{e}\left(u_{i}^{\prime}\right)\right)^{2}
$$

where $h$ refers to a certain distance class, $u_{i}$ and $u_{i}^{\prime}$ denote any two quadrats whose centers are separated by a distance belonging to the distance class considered, and $A(h)$ is the number of pairs of such quadrats. In addition, the semivariance was also estimated as a function of distance in four directions: at $0^{\circ}, 45^{\circ}, 90^{\circ}$, and $135^{\circ}$ from the within-row direction, with $90^{\circ}$ representing the acrossrow direction. In this case, only the pairs of observations (i.e., CRAD first-phase residuals) in the direction considered were included in the computation of the semivariance estimator. Variogram ordinates that start low and increase with increasing distance lag are a sign of the presence of spatial autocorrelation. The distance lag at which the semivariance reaches a plateau (i.e., a constant value) is called "range", and indicates the distance beyond which observations are no longer spatially correlated. When the plateau can only be reached asymptotically, the range is said to be "practical", and then corresponds to the distance at which the semivariance is equal to $95 \%$ of the plateau. Other important variogram model parameters are the nugget effect (i.e., the variogram ordinate at infinitely small distance) and the partial sill (measured as the difference between the height of the plateau and the nugget effect).

Because the spherical variogram model provides a good compromise between the Gaussian and exponential models in terms of rate of decrease of spatial autocorrelation with distance (10), the spherical model was chosen for fitting:

$$
\gamma(h)=c+b\left(1.5 \frac{h}{a}-0.5 \frac{h^{3}}{a^{3}}\right) \text { for } 0<h<a
$$

where $c$ represents the nugget effect, $b$ is the sill associated with the spherical variogram model, and $a$ denotes the range of spatial autocorrelation. The fitting of spherical variogram models to experimental variograms was performed by EGLS (43). Anisotropy was assessed by comparing the variogram model parameter estimates (i.e., range, nugget effect, and partial sill) obtained in the four directions and with the corresponding omnidirectional variogram.

Distributional analyses. The index of dispersion, $D$, was used to assess departure from complete randomness on each count data set. This index is a ratio of two variances: the observed variance of SNP incidence in the numerator and the variance of SNP incidence, estimated under the assumption of a binomial distribution, in the denominator:

$$
D=\frac{s_{y}^{2}}{\hat{p}(1-\hat{p}) / n}
$$

where $\hat{p}$ is the estimated probability (empirical frequency) of presence of a SNP. The general rule of interpretation is that a value of $D=1.0$ (i.e., the two variances are equal) indicates a completely random pattern within a sampling unit (i.e., a quadrat). A value of $D>1.0$ is indicative of a departure from a completely random pattern and is suggestive of heterogeneity. Under the assumption of a completely random spatial distribution, $(N-1) D$ follows a $\chi^{2}$ distribution with $N-1$ degrees of freedom, where $N$ is the sample size (i.e., number of quadrats) (31).

Binomial and $\beta$-binomial distributions were fitted to the frequency distributions of each count data set, using a SAS code developed by L. V. Madden and obtained from http://www. oardc.ohio-state.edu/pp702/Downloads.htm. When the location of an isolate carrying a given SNP is independent of the location of another isolate carrying the same SNP, the probability $p$ of an isolate carrying the SNP is constant over the field and the number $X$ of isolates carrying the SNP, out of $n$ isolates randomly sampled, follows a binomial distribution characterized by the probability function:

$$
\mathrm{P}(X=x)=\frac{n !}{x !(n-x) !} p^{x}(1-p)^{n-x} \quad x=0,1,2, \ldots, n
$$

The theoretical mean and variance of $X$ are equal to $n p$ and $n p(1-$ $p$ ), respectively. Compared with the binomial distribution $(X)$, the $\beta$-binomial distribution $(Y)$ has a supplementary parameter, $\theta$, used as a measure of local aggregation in space, and its probability function changes for:

$$
\mathrm{P}(Y=y)=\frac{n !}{y !(n-y) !} \frac{\prod_{j=0}^{y-1}(p+j \theta) \prod_{j=0}^{n-y-1}(1-p+j \theta)}{\prod_{j=0}^{n-1}(1+j \theta)}
$$

It follows that the theoretical mean and variance of $Y$ are $n p$ and $n p(1-p)(1+n \theta) /(1+\theta)$, respectively. Thus, the theoretical $\beta$ binomial variance can be seen as the product of the theoretical binomial variance $[n p(1-p)]$ and a heterogeneity component [(1 $+n \theta) /(1+\theta)]$. Accordingly, when local aggregation increases, the theoretical $\beta$-binomial variance is inflated with increasing $\theta$ value; in contrast, as $\theta$ approaches 0 , the $\beta$-binomial distribution approaches the binomial distribution $(16,26,27,31)$. Therefore, a good fit of the $\beta$-binomial distribution (i.e., the $\theta$ estimate is far from zero) indicates a departure from a completely random pattern, which means local aggregation of isolates carrying the same SNP within a quadrat in our study.

A likelihood-ratio test was performed to determine whether the $\beta$-binomial distribution provided a better fit to the observed frequencies of count data than the binomial distribution. The $\chi^{2}$ test statistics of goodness-of-fit were computed for the $\beta$-binomial and binomial distributions. Finally, a $C(\alpha)$ test based on the $z$ statistic was also used to determine whether the aggregation in the spatial distribution of each SNP was better described by a $\beta$ binomial distribution (26). The null hypothesis of our $\chi^{2}$ tests of goodness-of-fit was randomness, with aggregation as the alterna- 
tive hypothesis. The $C(\alpha)$ test is more specialized than the $\chi^{2}$ test because, even if the null hypothesis is the same, the alternative hypothesis of the former is more specific (i.e., that the $\beta$-binomial is appropriate) (48).

Sampling curves. Sampling curves were developed from the results obtained with the $\beta$-binomial distribution, so that the mean SNP incidence could be estimated with the "coefficient of variation of the sample mean" (31), denoted CV hereafter:

$$
\mathrm{CV}=\frac{\sqrt{\hat{p}(1-\hat{p})(1+\hat{\rho}(n-1)) / n N}}{\hat{p}}
$$

where $\hat{p}=\hat{\theta} /(\hat{\theta}+1), n$ is the number of subsamples (isolates) taken in each sampling unit (quadrat), and $N$ is the number of sampling units (number of quadrats). This equation can be rearranged to extract the sample size $N$ :

$$
N=\frac{(1-\hat{p})(1+\hat{\rho}(n-1))}{n \hat{p} \mathrm{CV}^{2}}
$$

The minimum number $N$ of sampling units needed to estimate $p$ with a given level of reliability was calculated from sampling curves developed for CV values of 10, 20, and 30\%. Sampling curves were calculated for three values of $\theta$ : the maximum, minimum, and median of the estimated values of $\theta$ for the $\beta$ binomial distributions fitted with our eight data sets $(28,31)$.

\section{RESULTS}

SNP incidence. The raw count data collected in this study are mapped in Figure 1 (see legend for the sample means and standard errors of the sample means). SNP incidence in $B$. cinerea populations was not consistently higher for one of the two vineyards, because the mean incidence is higher in the first vineyard (OR) for the H272R allele but higher in the second vineyard (TE) for the $1365 \mathrm{~S}$ and H272Y substitution. The mean incidence for the I365S substitution in B. squamosa populations was greater (in a ratio of 2.72) in 2010 than in 2009 (Fig. 1).

Geostatistical analyses. A drift (i.e., large-scale spatial component) was detected with the CRAD method in four of the eight data sets, accounting for 10.4 to $30.0 \%$ of the total variation (Table 1). After the removal of estimated drifts, spatial autocorrelation was found in six of the eight omnidirectional variograms of residual components. The estimated range of spatial autocorrelation varied from 11.9 to $28.7 \mathrm{~m}$, the mean being $19.9 \mathrm{~m}$ (Table 1). The nugget value tended to increase with increasing mean SNP incidence, whereas the sill of the spherical variogram model

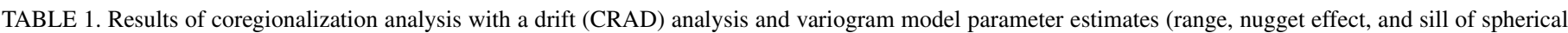

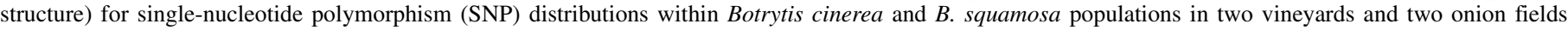

\begin{tabular}{|c|c|c|c|c|c|c|c|}
\hline Site & Year & SNP & Drift $^{\mathrm{a}}$ & Direction $\left({ }^{\circ}\right)^{\mathrm{b}}$ & Partial sill ${ }^{\mathrm{c}}$ & Nugget effect & Range of spatial autocorrelation (m) \\
\hline \multirow[t]{5}{*}{ OR } & 2011 & $\mathrm{H} 272 \mathrm{R}$ & 0 & Omni & 3.387 & 1.214 & 13.36 \\
\hline & & & & 0 & $\ldots$ & 4.734 & $\ldots$ \\
\hline & & & & 45 & 0.702 & 3.940 & 23.9 \\
\hline & & & & 90 & $\ldots$ & 4.200 & $\ldots$ \\
\hline & & & & 135 & $\ldots$ & 4.478 & $\ldots$ \\
\hline \multirow[t]{5}{*}{ OR } & 2011 & $\mathrm{H} 272 \mathrm{Y}$ & 0 & Omni & 0.969 & 2.406 & 22.96 \\
\hline & & & & 0 & 1.308 & 1.989 & 21.1 \\
\hline & & & & 45 & 0.579 & 2.743 & 21 \\
\hline & & & & 90 & 1.292 & 2.030 & 21.5 \\
\hline & & & & 135 & 1.161 & 2.045 & 20 \\
\hline \multirow[t]{5}{*}{ OR } & 2011 & $\mathrm{I} 365 \mathrm{~S}$ & 0.253 & Omni & 1.349 & 2.863 & 21.52 \\
\hline & & & & 0 & 1.837 & 2.258 & 21 \\
\hline & & & & 45 & $\ldots$ & 4.000 & $\ldots$ \\
\hline & & & & 90 & 0.962 & 3.065 & 21.5 \\
\hline & & & & 135 & 1.417 & 2.667 & 21.1 \\
\hline \multirow[t]{5}{*}{$\mathrm{DE}$} & 2009 & $\mathrm{I} 365 \mathrm{~S}$ & 0 & Omni & 4.089 & 0 & 12.88 \\
\hline & & & & 0 & $\ldots$ & 4.073 & $\ldots$ \\
\hline & & & & 45 & $\ldots$ & 3.914 & $\ldots$ \\
\hline & & & & 90 & $\ldots$ & 4.108 & $\ldots$ \\
\hline & & & & 135 & $\ldots$ & 3.864 & $\ldots$ \\
\hline \multirow[t]{5}{*}{ TE } & 2011 & H272R & 0.301 & Omni & 1.601 & 4.041 & 27.76 \\
\hline & & & & 0 & $\ldots$ & 5.441 & $\ldots$ \\
\hline & & & & 45 & 1.093 & 4.273 & 20.8 \\
\hline & & & & 90 & 3.146 & 2.469 & 21.3 \\
\hline & & & & 135 & 0.960 & 4.379 & 20.2 \\
\hline \multirow[t]{5}{*}{$\mathrm{TE}$} & 2011 & $\mathrm{H} 272 \mathrm{Y}$ & 0.23 & Omni & 2.668 & 2.349 & 28.72 \\
\hline & & & & 0 & 2.517 & 2.482 & 33.3 \\
\hline & & & & 45 & 3.494 & 1.368 & 20.7 \\
\hline & & & & 90 & $\ldots$ & 4.854 & $\ldots$ \\
\hline & & & & 135 & 2.150 & 2.444 & 20.4 \\
\hline \multirow[t]{5}{*}{$\mathrm{TE}$} & 2011 & $\mathrm{I} 365 \mathrm{~S}$ & 0 & Omni & 3.941 & 2.275 & 11.92 \\
\hline & & & & 0 & $\ldots$ & 6.420 & $\ldots$ \\
\hline & & & & 45 & 0.355 & 6.152 & 31.3 \\
\hline & & & & 90 & 0.470 & 5.928 & 21.5 \\
\hline & & & & 135 & 0.426 & 6.053 & 21.1 \\
\hline \multirow[t]{5}{*}{ VH } & 2010 & $\mathrm{I} 365 \mathrm{~S}$ & 0.104 & Omni & 0 & 4.763 & $\ldots$ \\
\hline & & & & 0 & 0.445 & 4.699 & 56.6 \\
\hline & & & & 45 & $\ldots$ & 4.531 & $\ldots$ \\
\hline & & & & 90 & 0.032 & 4.6 & 30.2 \\
\hline & & & & 135 & $\ldots$ & 4.739 & $\ldots$ \\
\hline
\end{tabular}
sampled in 2009 to 2011

\footnotetext{
a Drift estimated by estimated generalized least squares in first phase of CRAD (44).

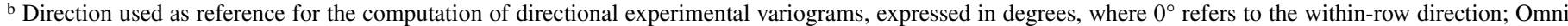
means that the experimental variograms were computed in all directions (i.e., they are omnidirectional).

c The sill (i.e., variance component) of the spherical structure used in variogram modeling.
} 
remained relatively small in general, implying that a moderate portion of the variance was explained by a spatially autocorrelated structure. Furthermore, directional variograms showed a great variety of spatial dependency, from acceptable isotropy in spatial autocorrelation (i.e., H272Y for OR in 2011) to various patterns of anisotropy, including the presence of spatial autocorrelation in only one direction (i.e., H272R for OR in 2011) and a large difference in the estimated range with direction (i.e., I365S for VH in 2010) (Table 1).

Spatial statistics. The value of the index of dispersion, $D$, was 1.71 to 2.97 , indicating a departure of medium-to-large magnitude of the frequency distribution of counts from a binomial distribution (Table 2). For all eight data sets, the maximum likelihood estimation procedure converged and the likelihood-ratio test showed that the $\beta$-binomial distribution provided a better fit to count data than the binomial distribution (Table 2). The $z$-value of the $\mathrm{C}(\alpha)$-test statistic was 5.16 to 14.44 with $P$ values of 0.0001 (Table 2), meaning there was local aggregation within quadrats, and this is well described with the $\beta$-binomial distribution (Fig. 2 ). The estimates of the $\beta$-binomial distribution parameters varied from 0.16 to 0.68 (average: 0.43 ) for $\hat{p}$ and from 0.09 to 0.23 (median: 0.20 ) for $\hat{\theta}$. The aggregation index values $(\hat{\theta})$ for

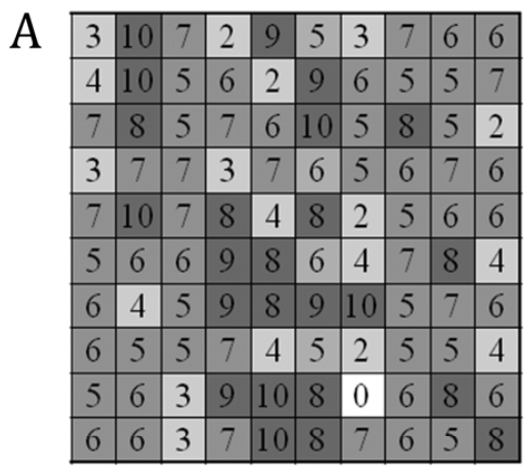

$\mathrm{B}$

\begin{tabular}{|l|l|l|l|l|l|l|l|l|l|}
\hline 6 & 0 & 1 & 0 & 1 & 5 & 4 & 2 & 0 & 2 \\
\hline 3 & 0 & 0 & 1 & 6 & 1 & 2 & 1 & 2 & 2 \\
\hline 0 & 2 & 4 & 3 & 2 & 0 & 1 & 0 & 2 & 3 \\
\hline 7 & 2 & 2 & 4 & 1 & 4 & 5 & 3 & 0 & 0 \\
\hline 3 & 0 & 0 & 0 & 3 & 2 & 8 & 1 & 0 & 3 \\
\hline 2 & 4 & 4 & 0 & 1 & 4 & 1 & 3 & 1 & 4 \\
\hline 3 & 1 & 4 & 0 & 1 & 1 & 0 & 3 & 1 & 2 \\
\hline 2 & 4 & 2 & 0 & 6 & 1 & 4 & 2 & 1 & 1 \\
\hline 3 & 2 & 3 & 0 & 0 & 2 & 0 & 0 & 1 & 2 \\
\hline 4 & 3 & 1 & 1 & 0 & 0 & 1 & 2 & 3 & 2 \\
\hline
\end{tabular}

C

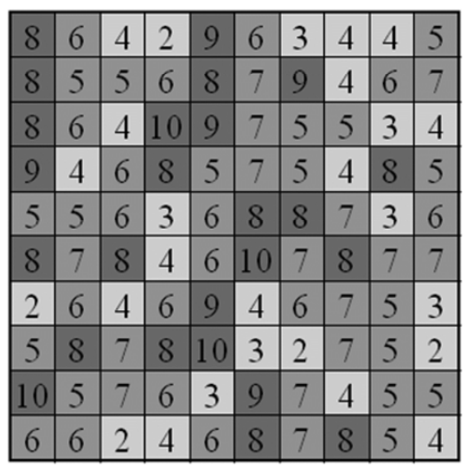

$\mathrm{D}$

\begin{tabular}{|l|l|l|l|l|l|l|l|l|l|}
\hline 5 & 5 & 9 & 10 & 9 & 4 & 7 & 6 & 3 & 1 \\
\hline 3 & 2 & 6 & 4 & 2 & 6 & 2 & 3 & 4 & 4 \\
\hline 5 & 1 & 5 & 6 & 2 & 2 & 5 & 3 & 6 & 4 \\
\hline 4 & 8 & 4 & 3 & 2 & 4 & 2 & 1 & 0 & 3 \\
\hline 1 & 0 & 0 & 1 & 1 & 4 & 3 & 2 & 5 & 3 \\
\hline 3 & 3 & 5 & 0 & 6 & 4 & 6 & 3 & 3 & 3 \\
\hline 3 & 2 & 3 & 9 & 0 & 4 & 4 & 2 & 2 & 3 \\
\hline 6 & 4 & 6 & 5 & 5 & 7 & 1 & 5 & 9 & 1 \\
\hline 4 & 3 & 3 & 1 & 7 & 4 & 6 & 3 & 8 & 8 \\
\hline 8 & 10 & 8 & 8 & 8 & 6 & 7 & 6 & 5 & 7 \\
\hline
\end{tabular}

$\mathrm{E}$

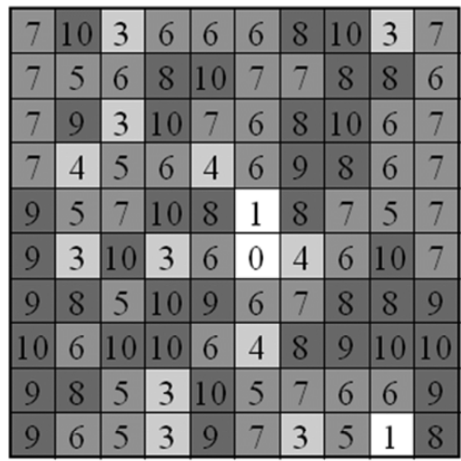

$\mathrm{F}$

\begin{tabular}{|l|l|l|l|l|l|l|l|l|l|}
\hline 2 & 1 & 2 & 4 & 2 & 1 & 3 & 1 & 0 & 0 \\
\hline 0 & 3 & 4 & 0 & 0 & 0 & 1 & 1 & 2 & 0 \\
\hline 1 & 0 & 0 & 1 & 4 & 0 & 2 & 3 & 3 & 4 \\
\hline 1 & 1 & 1 & 1 & 1 & 0 & 1 & 0 & 0 & 4 \\
\hline 0 & 0 & 0 & 0 & 6 & 1 & 5 & 0 & 3 & 1 \\
\hline 2 & 2 & 2 & 1 & 3 & 0 & 0 & 0 & 0 & 6 \\
\hline 0 & 0 & 1 & 2 & 0 & 2 & 1 & 6 & 0 & 0 \\
\hline 0 & 0 & 3 & 1 & 1 & 1 & 0 & 3 & 6 & 2 \\
\hline 0 & 1 & 1 & 0 & 6 & 9 & 10 & 0 & 1 & 0 \\
\hline 0 & 2 & 2 & 1 & 1 & 1 & 0 & 2 & 0 & 2 \\
\hline
\end{tabular}

$\mathrm{G}$

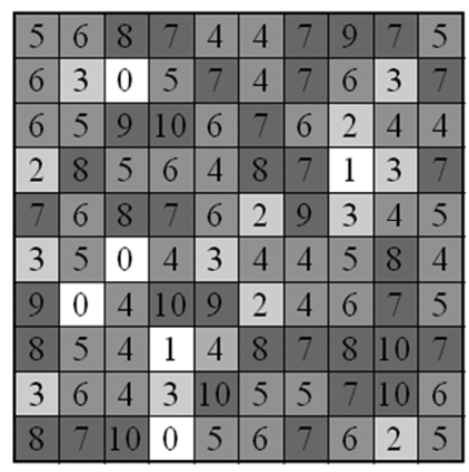

$\mathrm{H}$\begin{tabular}{l|l|l|l|l|l|l|l|l|l|}
\hline 2 & 1 & 2 & 4 & 2 & 1 & 3 & 1 & 0 & 0 \\
\hline & 0 & 3 & 4 & 0 & 0 & 0 & 1 &
\end{tabular}

\begin{tabular}{|c|c|c|c|c|c|c|c|c|c|}
\hline 0 & 3 & 4 & 0 & 0 & 0 & 1 & & 2 & 0 \\
\hline 1 & 0 & 0 & 1 & 4 & 0 & 2 & 3 & 3 & \\
\hline 1 & 1 & 1 & 1 & 1 & 0 & 1 & 0 & 0 & \\
\hline 0 & 0 & 0 & 0 & 6 & 1 & 5 & 0 & 3 & \\
\hline 2 & 2 & 2 & 1 & 3 & 0 & 0 & 0 & 0 & 6 \\
\hline 0 & 0 & 1 & 2 & 0 & 2 & 1 & 6 & 0 & 0 \\
\hline 0 & 0 & 3 & 1 & 1 & 1 & 0 & 3 & 6 & \\
\hline 0 & 1 & 1 & 0 & 6 & 9 & 10 & 0 & 1 & \\
\hline & 2 & 2 & & & & & & 0 & \\
\hline
\end{tabular}

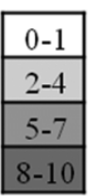

Fig. 1. Raw count data maps for Botrytis cinerea in vineyard OR for A, H272R (observed mean incidence, $0.68 \pm 0.023$, standard error); B, H272Y ( $0.21 \pm 0.021$ ); and C, I365S ( $0.55 \pm 0.024)$; and in vineyard TE for E, H272R (0.61 \pm 0.021$)$; F, H272Y (0.20 \pm 0.018$)$; and G, I365S ( $0.59 \pm 0.020)$; and for B. squamosa for I365S homologous in $\mathbf{D}, 2009(0.16 \pm 0.020)$ and $\mathbf{H}, 2010(0.42 \pm 0.025)$. 
$\mathrm{H} 272 \mathrm{R}, \mathrm{H} 272 \mathrm{Y}$, and $\mathrm{I} 365 \mathrm{~S}$ in $B$. cinerea populations were all lower in vineyard OR than in vineyard TE (Table 2). In contrast, the difference between $\theta$ estimates for I365S in the B. squamosa populations of 2009 and 2010 was small (Table 2).

Sampling curves. The developed sampling curves are presented in Figure 3. For a lower CV (i.e., higher precision), the minimum sample size $N$ required that the estimate of the mean SNP incidence should be larger. For $\hat{\theta}=0.09$ (i.e., smallest $\theta$ estimate in our study), $\approx 153,38$, and 17 quadrats should be sampled, with 10 isolates from each, to estimate a mean SNP incidence of 0.1 with $\mathrm{CV}$ values of 10,20 , and $30 \%$, respectively; for $\hat{\theta}=0.20$ (median estimated value), the three numbers of quadrats increase to 223,56 , and 25 ; and, for $\hat{\theta}=0.23$ (greatest estimated value), to 243, 61, and 27 (Fig. 3). By comparison, for a mean SNP incidence of 0.4 (i.e., fourfold $>0.1$ ), the required number of sampling quadrats is much smaller: $\approx 26,7$, and 3 with $\hat{\theta}=0.09$ and 37,8 , and 5 with $\hat{\theta}=0.20$ for $C V$ values of 10,20 , and $30 \%$, respectively (Fig. 3).

\section{DISCUSSION}

Management strategies, including fungicide applications, exist either to delay disease onset or to level off disease progress. Among the limitations regarding fungicide efficacy, the development of resistance by target organisms is becoming a priority for growers, crop specialists, and manufacturers. Despite the progress made toward understanding fungicide resistance at the individual level, establishing the relationship between theoretical and practical resistance is tedious and our knowledge remains limited. Recent research on resistance mechanisms, discovery of SNPs related to fungicide resistance, and development of molecular detection tools enables the breakdown of global phenotypic resistance into genotypic resistance. When moving from classical phenotypic to genotypic resistance, several hypotheses regarding practical aspects of resistance can be tested readily. For numerous aspects of fungicide resistance epidemiology, acquiring knowledge about spatial distribution patterns is essential, especially for sampling. Consequently, the data from two vineyards and two onion fields sampled in our study in 2011 and 2009 to 2010, respectively, were used to characterize the spatial distribution patterns of SNPs related to fungicide resistance in $B$. cinerea and B. squamosa populations.

In both systems, sampling was carried out toward the end of the growing season, after numerous secondary disease cycles; hence, diseased onion leaves or grape berries were found in each of the quadrats (5). However, within an onion field or a vineyard, the results of our geostatistical analyses suggest that quadrat counts on SNPs related to fungicide resistance are spatially autocorrelated, the estimated range of spatial autocorrelation varying between 21.0 and $31.7 \mathrm{~m}$ in omnidirectional analyses. The spatial autocorrelation measured was largely dependent on quadrat size and the organisms concerned in plant diseases. For example, Larkin et al. (22) found an average range of spatial autocorrelation of $\approx 15 \mathrm{~m}$ for Phytophthora capsici in bell pepper in soil and soil water. For almond leaf scorch disease, the estimated ranges of spatial autocorrelation averaged at $28 \mathrm{~m} \mathrm{(15)} \mathrm{and,} \mathrm{for}$ Venturia inequalis, the range of autocorrelation for potential ascospore dose was $40 \mathrm{~m}$ on average (6). In addition to relatively short ranges of spatial autocorrelation, our results indicated the presence of some anisotropy, which occurred in particular in the onion field sampled in 2010 and was then characterized by spatial autocorrelation restricted to the within-row direction and the direction perpendicular to it and by a much longer range of spatial autocorrelation in the former direction. These results support those of Elmer et al. (11), who also found spatial autocorrelation in the within-row direction in dicarboximide-resistant isolates from $M$. fructicola populations in stone fruit. In the two vineyards sampled in our study, the vines were planted $0.9 \mathrm{~m}$ apart on rows separated by $3 \mathrm{~m}$, thus forming a compact hedge. The presence of such hedges could explain the presence of a directional bias, which was moderate here. In the onion field with an anisotropic spatial autocorrelation in quadrat counts on SNPs related to fungicide resistance in 2010, the canopy height was lower and the crop density was much higher. It is unclear why the other onion field sampled the year before did not show a similar anisotropy, and actually did not show any spatial autocorrelation at all in quadrat counts on SNPs related to fungicide resistance. It is true, however, that the high values of the nugget effect relative to the sills of spherical variogram models fitted for the 2010 onion data set indicate that only a small part of the total variance could be explained by a spatially autocorrelated component. In addition, a short range of spatial autocorrelation is a prerequisite to the fitting of a $\beta$-binomial distribution.

Both $B$. cinerea isolates carrying the H272R, H272Y, and I365S mutations in the vineyards sampled and the B. squamosa isolates carrying the I365S homologous mutation in the onion fields sampled were heterogeneously distributed among sampling quadrats. The median estimated value of the parameter $\theta$ of the $\beta$ binomial distributions fitted to the eight-count data sets (Table 2) was 0.20 , indicating a relatively high level of small-scale spatial (i.e., local) aggregation. In fact, the $\beta$-binomial distribution provides an appropriate description of the frequency distribution of the number of mutations related to fungicide resistance per 10 isolates over sampling quadrats, the resistance status of a Botrytis

TABLE 2. Observed values of the index of dispersion and parameter estimates for the $\beta$-binomial and binomial distributions

\begin{tabular}{|c|c|c|c|c|c|c|c|c|c|c|c|c|c|}
\hline \multirow[b]{2}{*}{ Site } & \multirow[b]{2}{*}{ Year } & \multirow[b]{2}{*}{ SNP } & \multirow[b]{2}{*}{$\mathrm{MI}^{\mathrm{a}}$} & \multirow[b]{2}{*}{$D^{\mathrm{b}}$} & \multicolumn{2}{|c|}{$\mathrm{C}(\alpha)$ test $^{\mathrm{c}}$} & \multicolumn{2}{|c|}{ Parameter estimates $^{\mathrm{d}}$} & \multirow[b]{2}{*}{$\mathrm{LRT}^{\mathrm{e}}$} & \multirow[b]{2}{*}{$\chi^{2}{ }_{B B D}{ }^{f}$} & \multirow[b]{2}{*}{$P\left(\chi^{2}{ }_{\mathrm{BBD}}\right)^{\mathrm{f}}$} & \multirow[b]{2}{*}{$\chi^{2}{ }^{\mathrm{BD}^{\mathrm{g}}}$} & \multirow[b]{2}{*}{$P\left(\chi^{2}{ }_{\mathrm{BD}}\right)^{2}$} \\
\hline & & & & & $z$ & $P(z)$ & $\hat{p}(\mathrm{SE})$ & $\widehat{\theta}(\mathrm{SE})$ & & & & & \\
\hline OR & 2011 & $\mathrm{H} 272 \mathrm{R}$ & 0.607 & $1.87 *$ & 6.37 & 0.0001 & $0.6076(0.02103)$ & $0.1107(0.03048)$ & $28.72 * * *$ & 5.30 & 0.380 & 15.66 & 0.030 \\
\hline OR & 2011 & $\mathrm{H} 272 \mathrm{Y}$ & 0.197 & $1.96^{*}$ & 7.01 & 0.0001 & $0.1969(0.01713)$ & $0.1185(0.03390)$ & $31.06 * * *$ & 2.08 & 0.555 & 25.11 & 0.0001 \\
\hline OR & 2011 & $\mathrm{I} 365 \mathrm{~S}$ & 0.593 & $1.71 *$ & 5.16 & 0.0001 & $0.5936(0.02020)$ & $0.0851(0.02709)$ & $19.69 * * *$ & 1.05 & 0.975 & 17.51 & 0.002 \\
\hline TE & 2011 & $\mathrm{H} 272 \mathrm{R}$ & 0.684 & $2.49^{*}$ & 10.94 & 0.0001 & $0.6843(0.02306)$ & $0.2056(0.04645)$ & $68.89 * * *$ & 8.55 & 0.200 & 30.40 & 0.0001 \\
\hline TE & 2011 & $\mathrm{H} 272 \mathrm{Y}$ & 0.211 & $2.64 *$ & 12.06 & 0.0001 & $0.2135(0.01976)$ & $0.2067(0.04766)$ & $69.95 * * *$ & 2.06 & 0.725 & 39.14 & 0.0001 \\
\hline TE & 2011 & $\mathrm{I} 365 \mathrm{~S}$ & 0.550 & $2.42 *$ & 10.40 & 0.0001 & $0.5494(0.02434)$ & $0.1934(0.04145)$ & $65.00 * * *$ & 3.39 & 0.690 & 18.22 & 0.001 \\
\hline VH & 2010 & $\mathrm{I} 365 \mathrm{~S}$ & 0.422 & $2.51 *$ & 11.10 & 0.0001 & $0.4238(0.02451)$ & $0.1979(0.04369)$ & $70.05 * * *$ & 4.54 & 0.604 & 24.45 & 0.0001 \\
\hline $\mathrm{DE}$ & 2009 & $\mathrm{I} 365 \mathrm{~S}$ & 0.155 & $2.97 *$ & 14.44 & 0.0001 & $0.1592(0.01760)$ & $0.2335(0.05449)$ & $79.85 * * *$ & 4.41 & 0.219 & 39.65 & 0.0001 \\
\hline
\end{tabular}

a Mean incidence.

b Index of dispersion; * indicates significant at 0.05 level.

${ }^{c}$ Observed values of the $z$ statistic and corresponding probabilities of significance.

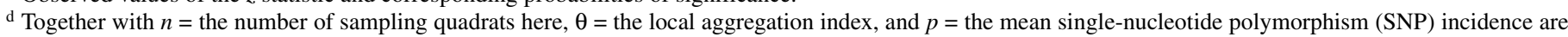
the three parameters of the $\beta$-binomial distribution; the binomial distribution has only two parameters, $n$ and $p$. Standard errors (SEs) are reported in parentheses.

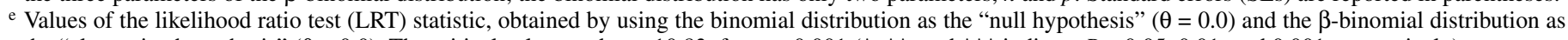
the "alternative hypothesis" $(\theta>0.0)$. The critical value used was 10.83 , for $\alpha=0.001(*, * *$, and *** indicate $P<0.05,0.01$, and 0.001 , respectively).

${ }^{\mathrm{f}}$ Observed values of the goodness-of-fit $\chi^{2}$ test statistic for the $\beta$-binomial distribution (BDD) and corresponding probabilities of significance.

g Observed values of the goodness-of-fit $\chi^{2}$ test statistic for the binomial distribution (BD) and corresponding probabilities of significance. 
isolate in a sampling quadrat being dependent on the resistance status of another Botrytis isolate in the same sampling quadrat. Using Lloyd's patchiness index, Elmer et al. (11) found that dicarboximide-resistant strains of $M$. fructicola in stone fruit were aggregated in all blocks and all years. Because very few studies have been conducted on the spatial distribution of resistant phenotypes or genotypes, the quantitative results obtained in our study are difficult to compare. However, the spatial aggregation characteristics of white mold epidemics caused by Sclerotinia spp. in bean $\left(\hat{\theta}_{\text {median }}=0.10\right)$ and pyrethrum $\left(\hat{\theta}_{\text {median }}=0.16\right)$, of leaf spot in strawberry $\left(\hat{\theta}_{\text {median }}=0.20\right)$, and of grape downy mildew (0.02 to 0.21$)$ were lower or similar $(19,29,45,49)$. For $B$.
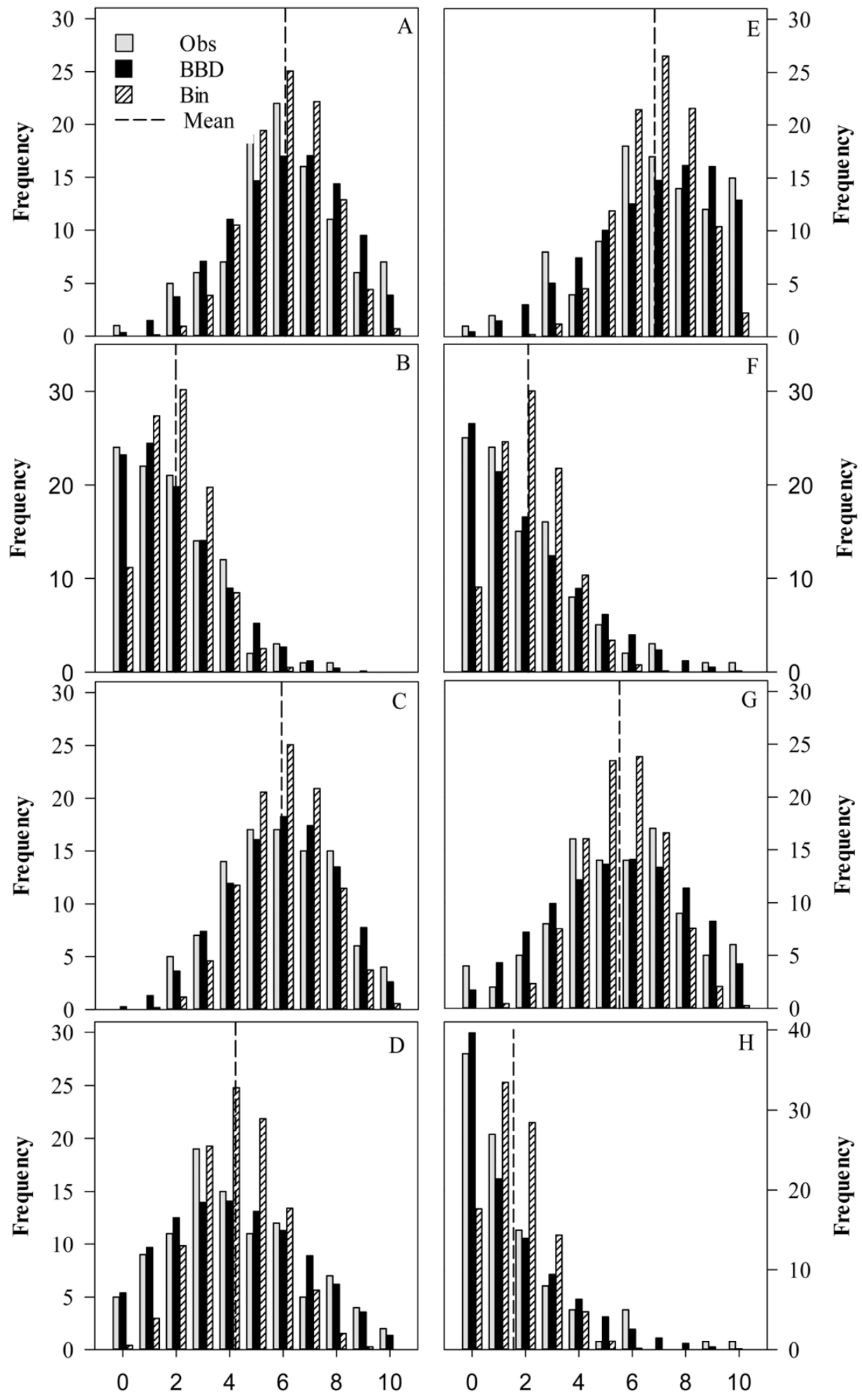

Number of resistant isolates

Number of resistant isolates

Fig. 2. Frequency distribution of the observed number of Botrytis isolates carrying a given single-nucleotide polymorphism (SNP) per quadrat (empty bars), together with the expected numbers of Botrytis isolates carrying the SNP per quadrat under the $\beta$-binomial distribution model (filled bars) and the binomial distribution model (dashed bars), for B. cinerea in vineyard OR for A, H272R; B, H272Y; and C, I365S; in vineyard TE for E, H272R; F, H272Y; and G, I365S; and for B. squamosa for I365S in D, 2009 and H, 2010. 
squamosa, the heterogeneity in the spatial distribution was well described with Iwao's patchiness regression technique (3). In that study, the authors concluded that the basic components of the Botrytis leaf blight lesion population were aggregates, contagiously distributed in the onion fields (3). The results reported here are suggestive of a small-scale dispersal pattern of genetic variants (i.e., the individuals carrying, or not, SNPs related to resistance), by opposition to the dispersal of a well-disbursed pathogen characterized by a completely random spatial distribution pattern (51). Spatial aggregation of plant diseases is predominantly determined by spore formation and dispersal. Consequently, if resistance to a given fungicide is associated with reduced sporulation, spore survival, or infection ability, the spatial distribution of resistant isolates is expected to be aggregated. However, recent studies by Parnell et al. $(41,42)$ have demonstrated that invasion is not the only possible outcome and various levels of co-existence between resistant and sensitive strains can be observed over a prolonged period of time in a mixed fungal population. Small-scale distribution can also be explained, in part, by the presence of autoinfection mechanisms, which are known to result in local clustering (36). The results of this study suggest that Botrytis strains carrying one or a series of SNPs may be considered as a subpopulation with different spatial behaviors.

Using the cluster sampling methodology described by Hughes et al. (18), we calculated the minimum sample sizes required to estimate mean SNP incidence with given levels of statistical precision and spatial aggregation. It was found (Fig. 3) that a larger sample size is required when the SNP follows an aggregated spatial distribution, compared with a completely random one. In other studies aimed to survey fungicide resistance using classical phenotypic assays, the sample size often comprised 10 to 100 samples per sampling site $(20,21,23,40)$. Sampling for fungicide resistance may not or should not require the same numbers of samples needed if resistance is tested by classical phenotypic assays or by genotypic assays, because the classical method encompasses all the genotypic variations. In fact, sampling for SNPs related to fungicide resistance requires a high level of precision. It might also be more extensive. For example, sampling for SNPs could be used for different purposes, such as day-to-day monitoring and surveillance of a population shift. Thus, sample size and level of reliability could vary depending on the objectives of the survey.

Because of the clonal reproduction and haploid dispersion of Botrytis spp., the expected range of spatial autocorrelation might be longer than the ranges estimated in our study. These were obtained from count data, after removal of a drift when present, for a given size of sampling quadrat. Nevertheless, our results strongly suggest aggregation in the spatial distribution patterns of SNPs related to fungicide resistance, which has important implications for questions of sample size. The large number of samples required when high precision matters represents a possible limitation toward sampling capacities. New detection methods belonging to different approaches allowing the analysis of bulked samples, such as pyrosequencing-based techniques or spore sampling devices for the quantification of mixed populations, should then be considered. Finally, thresholds for the proportion of resistant isolates above which fungicide control is inadequate still need to be determined.

\section{ACKNOWLEDGMENTS}

This work was financially supported in part by Agriculture and AgriFood Canada through a DIAP project. The contributions of $\mathrm{H}$. Van der Heyden and L. Brodeur to this work were partially supported by the Compagnie de Recherche Phytodata Inc. We thank M. Tremblay for his constant support; and A. Lefebvre, A Levasseur, and M. Gobeil-Richard for their essential technical assistance.
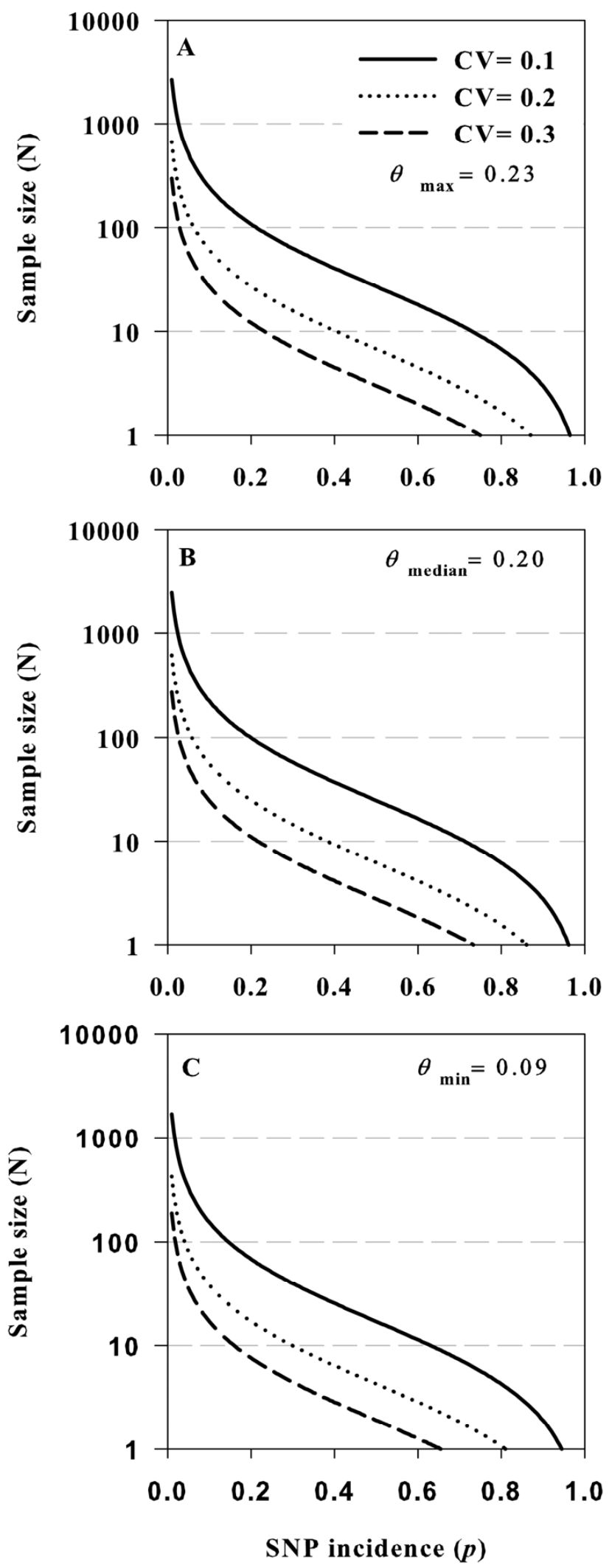

Fig. 3. Sampling curves calculated under the assumption of a $\beta$-binomial distribution, using low, intermediate, and high values of parameter $\theta$ that correspond to the minimum, median, and maximum values of $\theta$ estimates obtained in our study, for three levels of precision given by coefficient of variation of the sample mean (CV) values of 10,20 , and $30 \%$. Continuous, dotted, and dashed lines represent the minimum sample size required to estimate mean single-nucleotide polymorphism (SNP) incidence with a CV of 10,20 , and $30 \%$, respectively. 


\section{LITERATURE CITED}

1. Banno, S., Fukumori, F., Ichiishi, A., Okada, K., Uekusa, H., Kimura, M., and Fujimura, M. 2008. Genotyping of benzimidazole-resistant and dicarboximide-resistant mutations in Botrytis cinerea using real-time polymerase chain reaction assays. Phytopathology 98:397-404.

2. Billard, A., Laval, V., Fillinger, S., Leroux, P., Lachaise, H., Beffa, R., and Debieu, D. 2012. The allele-specific probe and primer amplification assay, a new real-time PCR method for fine quantification of singlenucleotide polymorphisms in pooled DNA. Appl. Environ. Microbiol. 78:1063-1068.

3. Boivin, G., and Sauriol, P. 1984. Dispersion statistics and sequential sampling plan for leaf blight caused by Botrytis squamosa in onions. Phytopathology 74:1385-1387.

4. Brent, K. J. and Hollomon, D. W. 2007. Fungicide Resistance: The Assessment of Risk, Second edition. Fungicide Resistance Action Committee. Monograph No. 2, GIFAP, Brussels.

5. Carisse, O., Savary, S., and Willocquet, L. 2008. Spatiotemporal relationships between disease development and airborne inoculum in unmanaged and managed Botrytis leaf blight epidemics. Phytopathology 98:38-44.

6. Charest, J., Dewdney, M., Paulitz, T., Philion, V., and Carisse, O. 2002. Spatial distribution of Venturia inaequalis airborne ascospores in orchards. Phytopathology 92:769-779.

7. Cui, W., Beever, R. E., Parkes, S. L., and Templeton, M. D. 2004. Evolution of an osmosensing histidine kinase in field strains of Botryotinia fuckeliana (Botrytis cinerea) in response to dicarboximide fungicide usage. Phytopathology 94:1129-1135.

8. Cui, W., Beever, R. E., Parkes, S. L., Weeds, P. L., and Templeton, M. D. 2002. An osmosensing histidine kinase mediates dicarboximide fungicide resistance in Botryotinia fuckeliana (Botrytis cinerea). Fungal Genet. Biol. 36:187-198.

9. Dallot, S., Gottwald, T., Labonne, G., and Quiot, J. B. 2003. Spatial pattern analysis of Sharka disease (Plum pox virus strain M) in peach orchards of southern France. Phytopathology 93:1543-1552.

10. Dutilleul, P. R. L. 2011. Spatio-Temporal Heterogeneity: Concept and Analyses. Cambridge University Press, New York.

11. Elmer, P. A. G., Gaunt, R. E., and Frampton, C. M. 1998. Spatial and temporal characteristics of dicarboximide-resistant strains of Monilinia fructicola and brown rot incidence in stone fruit. Plant Pathol. 47:530536.

12. Ferrandino, F. J. 2004. Measuring spatial aggregation in binary epidemics: correlative analysis and the advantage of fractal-based sampling. Phytopathology 94:1215-1227.

13. Fillinger, S., Leroux, P., Auclair, C., Barreau, C., Al Hajj, C., and Debieu, D. 2008. Genetic analysis of fenhexamid-resistant field isolates of the phytopathogenic fungus Botrytis cinerea. Antimicrob. Agents Chemother. 52:3933-3940.

14. Fournier, E., Levis, C., Fortini, D., Leroux, P., Giraud, T., and Brygoo, Y. 2003. Characterization of Bc-hch, the Botrytis cinerea homolog of the Neurospora crassa het-c vegetative incompatibility locus, and its use as a population marker. Mycologia 95:251-261.

15. Groves, R. L., Chen, J., Civerolo, E. L., Freeman, M. W., and Viveros, M. A. 2005. Spatial analysis of almond leaf scorch disease in the San Joaquin Valley of California: factors affecting pathogen distribution and spread. Plant Dis. 89:581-589.

16. Hughes, G., and Madden, L. V. 1993. Using the beta-binomial distribution to describe aggregated patterns of disease incidence. Phytopathology 83:759-763.

17. Hughes, G., and Madden, L. V. 1995. Some methods allowing for aggregated patterns of disease incidence in the analysis of data from designed experiments. Plant Pathol. 44:927-943.

18. Hughes, G., Madden, L. V., and Munkvold, G. P. 1996. Cluster sampling for disease incidence data. Phytopathology 86:132-137.

19. Jones, S. J., Gent, D. H., Pethybridge, S. J., and Hay, F. S. 2011. Spatial characteristics of white mould epidemics and the development of sequential sampling plans in Australian bean fields. Plant Pathol. 60:11691182.

20. Köller, W., Wilcox, W. F., Barnard, J., Jones, A. L., and Braun, P. G. 1997. Detection and quantification of resistance of Venturia inaequalis populations to sterol demethylation inhibitors. Phytopathology 87:184190.

21. Köller, W., Wilcox, W. F., and Jones, A. L. 1999. Quantification, persistence, and status of dodine resistance in New York and Michigan orchard populations of Venturia inaequalis. Plant Dis. 83:66-70.

22. Larkin, R. P., Gumpertz, M. L., and Ristaino, J. B. 1995. Geostatistical analysis of Phytophthora epidemic development in commercial bell pepper fields. Phytopathology 85:191-203.

23. Leroch, M., Kretschmer, M., and Hahn, M. 2011. Fungicide resistance phenotypes of Botrytis cinerea isolates from commercial vineyards in South West Germany. J. Phytopathol. 159:63-65.
24. Leroux, P., Fritz, R., Debieu, D., Albertini, C., Lanen, C., Bach, J., Gredt, M., and Chapeland, F. 2002. Mechanisms of resistance to fungicides in field strains of Botrytis cinerea. Pest Manage. Sci. 58:876-888.

25. Leroux, P., Gredt, M., Leroch, M., and Walker, A.-S. 2010. Exploring mechanisms of resistance to respiratory inhibitors in field strains of Botrytis cinerea, the causal agent of gray mold. Appl. Environ. Microbiol. 76(19):6615-6630.

26. Madden, L. V., and Hughes, G. 1994. BBD-computer software for fitting the beta-binomial distribution to disease incidence data. Plant Dis. 78:536-540.

27. Madden, L. V., and Hughes, G. 1995. Plant disease incidence: distributions, heterogeneity, and temporal analysis. Annu. Rev. Phytopathol. 33:529-564.

28. Madden, L. V., and Hughes, G. 1999. Sampling for plant disease incidence. Phytopathology 89:1088-1103.

29. Madden, L. V., Hughes, G., and Ellis, M. A. 1995. Spatial heterogeneity of the incidence of grape downy mildew. Phytopathology 85:269-275.

30. Madden, L. V., Hughes, G., and Munkvold, G. P. 1996. Plant disease incidence: inverse sampling, sequential sampling, and confidence intervals when observed mean incidence is zero. Crop Prot. 15:621-632.

31. Madden, L. V., Hughes, G., and van den Bosch, F. 2007. The Study of Plant Disease Epidemics. American Phytopathological Society Press, St. Paul, MN.

32. Madden, L. V., Nault, L. R., Murral, D. J., and Apelt, M. R. 1995. Spatial pattern analysis of the incidence of aster yellows disease in lettuce. Res. Pop. Ecol. 37:279-289.

33. Matheron, G. 1962. Traité de géostatistique appliquée. Vol. 1, Mem. Bur. Rech. Geol. Minières. No. 14, Editions Technip, Paris, France.

34. Mavroeidi, V. I., and Shaw, M. W. 2005. Sensitivity distributions and cross-resistance patterns of Mycosphaerella graminicola to fluquinconazole, prochloraz and azoxystrobin over a period of 9 years. Crop. Prot. 24:259-266.

35. Mavroeidi, V. I., and Shaw, M. W. 2006. Effects of fungicide dose and mixtures on selection for triazole resistance in Mycosphaerella graminicola under field conditions. Plant Pathol. 55:715-725.

36. Mundt, C. C. 2009. Importance of autoinfection to the epidemiology of polycyclic foliar disease. Phytopathology 99:1116-1120.

37. Myresiotis, C. K., Karaoglanidis, G. S., and Tzavella-Klonari, K. 2007. Resistance of Botrytis cinerea isolates from vegetable crops to anilinopyrimidine, phenylpyrrole, hydroxyanilide, benzimidazole, and dicarboximide fungicides. Plant Dis. 91:407-413.

38. Oshima, M., Banno, S., Okada, K., Takeuchi, T., Kimura, M., Ichiishi, A., Yamaguchi, I., and Fujimura, M. 2006. Survey of mutations of a histidine kinase gene BcOS1; in dicarboximide-resistant field isolates of Botrytis cinerea. J. Gen. Plant Pathol. 72:65-73.

39. Oshima, M., Fujimura, M., Banno, S., Hashimoto, C., Motoyama, T., Ichiishi, A., and Yamaguchi, I. 2002. A point mutation in the two-component histidine kinase $B c O S-1$ gene confers dicarboximide resistance in field isolates of Botrytis cinerea. Phytopathology 92:75-80.

40. Pappas, A. C. 1997. Evolution of fungicide resistance in Botrytis cinerea in protected crops in Greece. Crop. Prot. 16:257-263.

41. Parnell, S., Gilligan, C. A., and van den Bosch, F. 2005. Small-scale fungicide spray heterogeneity and the coexistence of resistant and sensitive pathogen strains. Phytopathology 95:632-639.

42. Parnell, S., van den Bosch, F., and Gilligan, C. A. 2006. Large-scale fungicide spray heterogeneity and the regional spread of resistant pathogen strains. Phytopathology 96:549-555.

43. Pelletier, B., Dutilleul, P., Larocque, G., and Fyles, J. 2004. Fitting the linear model of coregionalization by generalized least squares. Math. Geol. 36:323-343.

44. Pelletier, B., Dutilleul, P., Larocque, G., and Fyles, J. 2009. Coregionalization analysis with a drift for multi-scale assessment of spatial relationships between ecological variables 1. Estimation of drift and random components. Environ. Ecol. Stat. 16:439-466.

45. Pethybridge, S. J., Hay, F. S., and Gent, D. H. 2010. Characterization of the spatiotemporal attributes of Sclerotinia flower blight epidemics in a perennial pyrethrum pathosystem. Plant Dis. 94:1305-1313.

46. Saito, S., Suzuki, S., and Takayanagi, T. 2009. Nested PCR-RFLP is a high-speed method to detect fungicide-resistant Botrytis cinerea at an early growth stage of grapes. Pest Manage. Sci. 65:197-204.

47. Samuel, S., Papayiannis, L. C., Leroch, M., Veloukas, T., Hahn, M., and Karaoglanidis, G. S. 2011. Evaluation of the incidence of the G143A mutation and cytb intron presence in the cytochrome bc-1 gene conferring QoI resistance in Botrytis cinerea populations from several hosts. Pest Manage. Sci. 67:1029-1036.

48. Tarone, R. E. 1979. Testing the goodness of fit of the binomial distribution. Biometrika 66:585-590.

49. Turechek, W. W., and Madden, L. V. 1999. Spatial pattern analysis and sequential sampling for the incidence of leaf spot on strawberry in Ohio. Plant Dis. 83:992-1000. 
50. Turechek, W. W., and Madden, L. V. 2000. Analysis of the association between the incidence of two spatially aggregated foliar diseases of strawberry. Phytopathology 90:157-170.

51. Turechek, W. W., and Mahaffee, W. F. 2004. Spatial pattern analysis of hop powdery mildew in the Pacific Northwest: implications for sampling. Phytopathology 94:1116-1128.

52. Xiao, C. L., Hao, J. J., and Subbarao, K. V. 1997. Spatial patterns of microsclerotia of Verticillium dahliae in soil and Verticillium wilt of cauliflower. Phytopathology 87:325-331.

53. Yin, Y. N., Kim, Y. K., and Xiao, C. L. 2011. Molecular characterization of boscalid resistance in field isolates of Botrytis cinerea from apple. Phytopathology 101:986-995.

54. Ziogas, B. N., Nikou, D., Markoglou, A. N., Malandrakis, A. A., and Vontas, J. 2009. Identification of a novel point mutation in the $\beta$-tubulin gene of Botrytis cinerea; and detection of benzimidazole resistance by a diagnostic PCR-RFLP assay. Eur. J. Plant Pathol. 125:97-107. 